\title{
A Study on Music Genre Recognition and Classification Techniques
}

\author{
Aziz Nasridinov ${ }^{1}$ and Young-Ho Park $*^{2}$ \\ aziz@dongguk.ac.kr,yhpark@sm.ac.kr \\ *Corresponding Author
}

${ }^{1}$ School of Computer Engineering, Dongguk University at Gyeongju

${ }^{2}$ Department of Multimedia Science, Sookmyung Women's University

\begin{abstract}
Automatic classification of music genre is widely studted topic in music information retrieval (MIR) as it is an efficient method to structure and organize the large numbers of music files available on the Internet. Generally, the gen re classification process of music has two main steps: feature extraction and classification. The finst step obtains audio signal information, while the second one classifies the music into various genres according to extracted features. In this paper, we present astudy on techniques for automatic music genre recognition and classification. We firstedescribe machine learning based chord recognition methods, such as hidden Markov models, neurck netyorks, dynamic Bayesian network and rule-based methods, and template matching methods. We then explain supervised, unsupervised and semi-superyised classificotion methods classifying music genres. Finally, we briefly describe the proposed method for automatic classification of music genres, which consists of three steps. chond labeling, genre matching and classification.
\end{abstract}

Keywords: hord récognition, genre classification, subsequence matching, decision tree

\section{Introduction}

The rapid development of Internet together with the growth of the bandwidth availability have resulted in the widespread of large amounts of digital multimedia contents in Iriternet [1]. One of the most important types of multimedia content distributed ovey the Internet is a high volume of digital music in MP3 format. This has motivated researchers to develop music information retrieval (MIR) techniques that would be helpful for Internet music search engines, musicologist and listeners to find music from numerous options. Among these techniques, automatic classification of music pieces into categories such as mood, artist or genre is widely studied topic in MIR as it is an efficient method to structure and organize the large numbers of music files available on the Internet.

A music genre describes a style of music that has similar characteristics shared by its members and can be distinguished from other types of music. These characteristics are usually related to the instrumentation, rhythm, harmony, and melody of the music [2]. Generally, the genre classification process of music has two main steps: feature extraction and classification. The first step obtains audio signal information, while the second one classifies the music into various genres according to extracted features. Chord recognition methods are used for feature extraction from an audio signal. The 
chord recognition task constructs a chord label from a specific music-related feature. On the other hand, different data-mining algorithms, including supervised, unsupervised and semi-supervised classification, are proposed to classifying music genres.

In this paper, we present a study on techniques for automatic music genre recognition and classification. We first describe machine learning based chord recognition methods, such as hidden Markov models, neural networks, dynamic Bayesian network and rulebased methods, and template matching methods. We then explain supervised, unsupervised and semi-supervised classification methods classifying music genres. Finally, we briefly describe the proposed method for automatic classification of music genres, which consists of three steps: chord labeling, genre matching and classification.

The rest of the paper is organized as follows. In Section 2, we discuss techniques on music genre classification. In Section 3, we briefly describe the proposed method. In Section 4, we highlight conclusions and future work.

\section{Techniques on Music Genre Classification}

Generally, the genre classification process of music has two/main steps: feature extraction and classification. The first step obtains audio signa/information, while the second one classifies the music into various genres accordmg to extracted features. Chord recognition methods are used for feature extractionfrom an audio signal. Thus, in Section 2.1, we discuss chord recogaition methods. In Section 2.2, we explain music genre classification methods.

\subsection{Chord Recognition}

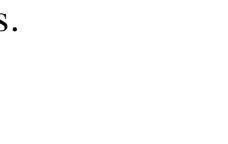

The chord recognition fask constructs a chord label from a specific music-related feature. Typically, chord reeognition techniques use a chromagram as an input to the system and output a chord label for each chromagram frame. Various methods have been used for this task. In this subsection, we discuss machine-learning methods and template matching methods.

2.1.1. Machine-learning based cord recognition: Hidden Markov models are the most popular methods used in chord recognition. One of the earliest implementation of such a method is described in [3]. In [3], the authors proposed to construct a system for automatic chord transcription using speech recognition tools. In the proposed method, input signal is first segmented to the frequency domain. Then, it is mapped to the Pitch Class Profile domain, where vectors are used as features to train a hidden Markov model with one state for each chord. These features are then used to construct chord models via ExpectationMaximization algorithm. Finally, chord recognition is carried out with the Viterbi algorithm. The experimental results with a small set of 20 songs demonstrate that the proposed method has a frame-level accuracy of around $75 \%$ on forced-alignment task.

In [4], the authors proposed a method for semantically describing harmonic content directly from music signals. In the proposed method, the audio signal is first transformed into tactus window and pitch chroma tuning process is done. Then, cord labeling is accomplished where a lexicon of 24 triads is used in order to describe harmonic movement in a piece. The hidden Markov model algorithm is then initialized that keeps dependency between tonic, mediant, and dominant pitches in a triad, along with the consonance between neighboring triads in a sequence. Finally, the updates to models parameters of hidden Markov model algorithm is performed in order to maintain the relationship between pitches in a chord.

In $[5,6]$, the authors proposed an automatic chord recognition method from audio using an hidden Markov model with supervised learning. For this, the authors used symbolic data to 
make label files and create audio files. For feature vectors, the authors used 12-bin tune chrome vectors. Each state in hidden Markov models was designed by a multivariate, single Gaussian completely represented by its mean vector and a diagonal covariance matrix. In their model, the authors used 36 chord types that include three distinct sonorities, such major, minor and diminished, for each pitch type. Once the model parameters, such as initial state probabilities, state transition probabilities, and mean vector and covariance matrix for each state, are estimated from the training data, the Viterbi algorithm is applied to the model to find the chord sequence from input signal. The proposed system is demonstrated in Figure 1. The results of experiments demonstrate that the proposed method has a higher performance in frame-level chord recognition than existing methods.

In [7], the authors proposed a simultaneous estimation of chord progression and downbeats from an audio file. In the proposed method, to extract chord progression and the downbeats from the audio signal, a set of meter-related feature vectors that describe the signal is extracted. The chord progression is described using a hidden Markov model that considers global dependencies on meter. In orded to extract the tactus/tatum positions, the authors used the method introduced in [8]. The proposed method is evaluated on a dataset of 66 popular music songs from the Beatles and shows improvement over the existing methods.

In [9], the authors proposed a method for automatic chord recognition using hidden Markov models. The applicability of standard factored languages to the chord recognition are also studied. In the proposed method, pitch type profile vectors are first extracted from the given audio signal. Then, irorder to capture chord sequence, Viterbi decoder on trained hidden Marko hodels and sabsequent lattice rescoring, applying the language model weight is used. The, experiment results with 175 manually-labelled songs demonstrate that the proposed method provided an increase in accuracy of about $2 \%$.

In [10], the authors proposed a chord recognition method using duration-explicit hidden Markov models. The moder break up the duration constraints from the transition matrix. Then, the method construdts distinct models for duration distributions that demonstrate time signatures to improve the duration constraint in each model. The proposed method is experimented using Uspop dataset and demonstrates that it recognizes chords withaceuracy to $84.23 \%$.

In [11], the aurhors argue that traditional hidden Markov model, with Vitebri algorithm, is not surtable for real-time chord recognition, and proposed a system of buffers and á modified decoding process that approximates offline results while minimizing the system's latency. The authors used two different types, such as an audio input buffer and an observation buffer. The audio input buffer estimates block size of audiosamples from incoming audio signals. Each time new hop size of samples comes into the buffer, chroma features are extracted from audio input buffer. For local decoding process, the observation buffer saves $L$ number of the extracted chroma vectors. The experiment results show that the real-time system can be as efficient as offline systems. 


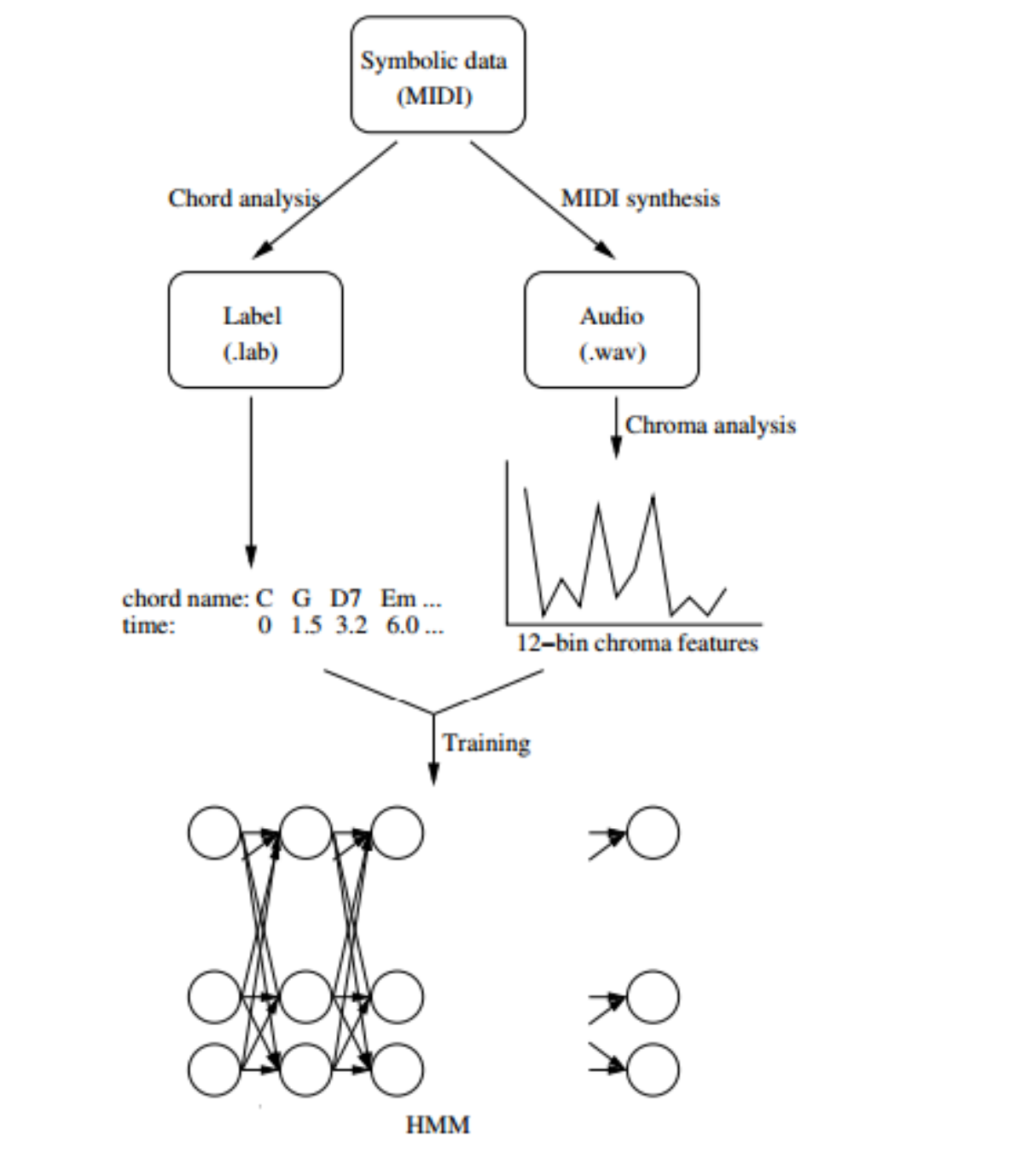

Figure 1. Automatic chord recognition from audio using hidden Markov model [5]

In order to speed up the process of chord recognition compared to hidden Markov model, some of the studies used neural networks. For example, in [12], the authors proposeda feed forward neural network method for chord recognition. Through various experimems, the authors demonstrate that using 12-dimensional Pitch Class Profile vectors are effective representation method of chords. Specifically, the experiment result demonstrate that the Pitch Class Profile is well-suited for describing chords in a machine learning context, and the algorithm is capable to recognize chords played with other instruments. In [13], the authors proposed an audio chord recognition system based on a recurrent neural network. The proposed model can learn fundamental musical properties, such as temporal continuity, temporal dynamics and harmony. The authors propose an algorithm that is able to search for the chord sequences when the audio signal is ambiguous, noisy or weakly discriminative. Through various experiments with MIREX dataset, the authors demonstrate that the proposed method is competitive with existing approaches. 
Another body of the literature used dynamic Bayesian network to recognize chords of the music. For example, in [14], the authors present dynamic Bayesian network, which integrates models of metric position, key, chord, bass note and two beat-synchronous audio features (bass and treble chroma) into a single high-level musical context model. With 109 chord types, the model provides a higher level of detail than existing approaches while maintain a high level of accuracy. In $[15,16]$, the authors proposed a modification to [14], which integrates in a single probabilistic model the hidden states of metric position, key, chord, and bass node, along with two observed variables: chroma and bass chroma.

Rule-based recognition methods are presented in [17, 18]. In [17], the authors proposed a framework to analyze a musical audio signal sampled from a popular music, and define its key, offer usable chord transcriptions, and extract the hievarchical rhythms structure representation including the quarter-note, half-note and whole note levels. In chord accuracy enhancement phase, the authors perform a rule-based analysis of the detected chord to check if it exists in the key of the song. In [18], the authors proposed a signal based approach to obtain chords from a music files. Information about chords are obtained from spectral representations of the audio data. Then, for each frequency bub in the desired frequency range, the amplitudes of all hypothetic harmonics are summarized. To extract the most salient pitches, the eight strongest local maxima in the resulting sum spectrum are chose as candidates. From this set of chord candidates, the most plausible sequence of chords sequence of chords is obtained using the following criteria: maximization of amplitade, maximization of duration and fitness to the detected key.

2.1.2. Template-based cord recognition: There are several methods that used different techniques to machine learning techniques to recognize chords of the music. Template based techniques are one of them and use pre-defined chord models to recognize the cord type. For example, in $19,20,21$, instead of using hidden Markov models, the authors proposed a chord recognition method using measures of fit, chord templates and filtering methods. In the proposed method, they first calculate chrome vectors from input audio signal. A set of chord templates for several types of chords are also proposed. A scale parameter is then calculated in order to fit the chroma vectors to the chord templates. The detected chord is the one minimizing the measure of fit between a rescaled chroma vector and the chord templates. In order to consider the timepersistence, the authors performed a post-processing filtering that can smooth the results and correct the errors.

In $[22,231$, the authors proposed a probabilistic template-based chord recognition. The key distinction of the proposed method to the existing template-based approach is that the authors proposed chord probabilities, learned from the song. The result of the proposed method demonstrate that there is no probability of spurious chords, which smoothed out the transcription produced with deterministic approach. The method is tested on dataset consists of the 180 pop-rock songs and the other one that is composed of 20 songs from various artists and music genres. The results of experiments show that the proposed method has better performance in chord recognition comparing to existing work.

In [24], the authors proposed a method for automatic chord recognition from audio files using enhanced pitch class profile. In order to do so, the authors introduced a feature vector called the Enhanced Pitch Class Profile (EPCP). In the proposed method, first, the Harmonic Product Spectrum is extracted from the Discrete Fourier transform of the input audio signal. An algorithm for calculating a 12-dimensional pitch class 
profile is then used to it to provide the EPCP feature vector. The EPCP vector is correlated with pre-determined templates for 24 major/minor triads, and the template with maximum correlation is identified as the chord of the input audio signal. The experiment results demonstrate that the EPCP is error-prone compared to the traditional PCP in frame-rate chord recognition.

\subsection{Genre Classification}

One of the most important types of multimedia data distributed over the Web is a high volume of digital music in MP3 format. Music genre classification is effective way to structure and organize the large numbers of music files on the Web. Several methods have been used for this task. In this subsection, we discuss the supervised, unsupervised and semi-supervised classification methods.

2.2.1. The supervised classification method: In supervis ed method $\widehat{s}$, the-model is first trained by manually labeled data, i.e., supervised classification knows the genres of songs. In [25], the authors proposed a music gemreclassification method using multilayer support vector machine learning. In this method, to characterize music content, beat spectrum, linear prediction coefficients, zero crossing rates, short time energy and mel-frequency cepstral coefficients are used as features. Support vector machines are developed to obtain optimal class boundaries between different kinds of music genres by learning from training data. Through various experiments, the authors demonstrated that multi-layer support vector machines have better performance compared to traditional Euclidean distance bâsed methods and statistical learning methods.

In [26], the authors explore automatic classification of audio signals into a hierarchy of musical genres. The main focus of the proposed method is to discover three feature sets for representing timbral texture, rhythmic content and pitch content. Gaussian mixture model (GMM) and $\mathrm{K}$-nearest neighbor (KNN) classifiers are used based on the extracted features. Through experiments, the authors show that the proposed method has accuracy of $61 \%$ (non-rea time) and $44 \%$ (real time) when there are ten musical genres present.

2.2.2. The unsupervised classification method: In contrast to supervised classification methods, unsupervised dlassification methods classify data based on no knowledge about the genre clasters. In [2], the authors argue that supervised classification methods may not be appropriate for personal music management as manually labeling music for each individual-defined genres can be difficult and inconsistent. They proposed to partition a music genres into several clusters by measuring the similarities between musie based on cross likelihood ratio, inverse Euclidean norm, and cosine measure. Nex. the authors used hierarchical agglomerative clustering in order to group music of one genre. A method based on Rand index is also proposed to define the optimal number of clusters automatically according to the number of genres. The experiment results show the feasibility of proposed method in music genre classification.

In [27], the authors proposed an unsupervised learning method of local features. In the proposed method, local patches from the time-frequency transformed audio signal is obtained. It is then pre-processed by normalizing all patches and applying whitening transformation to the patches. Furthermore, unsupervised learning of an overcomplete dictionary of local features is used. For learning, the authors used earthier bootstrapped $k$-means algorithm or select features randomly. Feature responses are obtained in a convolutional manner and trained using a linear support vector machine for 
classification. Through various experiments, it is demonstrated that the proposed method is competitive with existing classification methods.

In [28], the authors proposed a method to classify music genres using hidden Markov models. The proposed method contains two steps. In the first step, to characterize music content, segmentation scheme is used based on music intrinsic rhythmic structure analysis as features. Then, based on these features, hidden Markov model is trained for the music piece. In the second step, a distance matrix is constructed of the distance between every pair of music pieces (hidden Markov models) and clustering to make desired clusters are performed. The authors experimentally show that the proposed method performs as good as supervised classification methods.

2.2.3. The semi-supervised classification method: The semi-supervised cfassification methods make use of both supervised and unsupervised classification methods. That is a small amount of labeled data with a large amount of unlabeled data are used for training. In [29], the authors proposed a semi-supervised classiffcation method for musical genres using multi-view features. The method proposes to combine/different feature sets, such as Short Time Fourier Transform (STFT) based featyres, Mel Frequency Ceptral Coefficients (MFCC) features, and Discrete Wavelet Transform (DWT) based features, and split it into several feature subsets. Co-Training algorithm is then applied to classify music pieces with only few annotations compared to traditional supervised classification methods that need large amount of annotations. Through various experiments, the authors prove the validity and eifectiveness of the proposed method.

In [30], the authors proposed aframework for hierarchical music classification of music pieces into a genre taxonomy. A music piece is described by a set of feature vectors, where number of instances can be from tens to hundreds per song. Thus, in the proposed method, the auth frs first introduce hierarchical semi-supervised technique for instance reduction. This reductions are then used for hierarchical classification music pieces with support vector machines. Furthermore, the authors use object adjusted weighting in order to take advantage from multiple representations. Experiment results with real data set demonstrate that the proposed method is efficient has high classification accuracy.

\section{Proposed Method}

In this section, we briefly describe the proposed method. Our method consists of three main steps. In Section 3.1, we describe the chord labeling scheme. In Section 3.2, we explain the genre matching process. In Section 3.3, we introduce the genre classification.

\subsection{Chord Labeling Scheme}

In the proposed method, we first annotate the music sequence using the binary sequence. Figure 1 demonstrates the example of chord labeling scheme, where a "1" is used to denote a sound that is going up in the music sequence, and a " 0 " is used to represent a sound that is going down. Note that a sound that does not change it is state in the music sequence is denotes as " 0 ". Thus, the chord labeling scheme for a music sequence presented in Figure 2 is as following: [11010101]. 


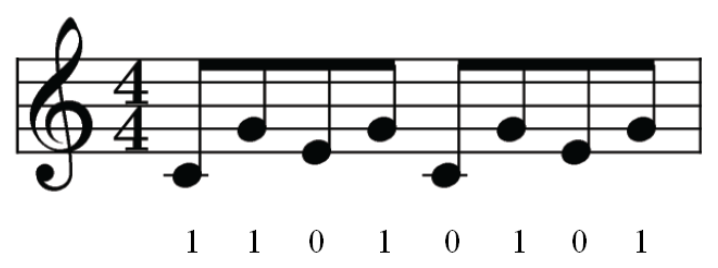

Figure 2. Representation of music sequence using the binary sequence.

\subsection{Genre Matching}

Once the music sequence is demoted with binary sequence, we can consider it as time sequence and find the subsequence matching to the genre. Given two time sequence $S$ and $Q$ which may have different length, the goal is to find all similar subsequence pairs between two time sequences with a specified threshold [31]. A $k$-windowSugsequence Alatching algorithm, described in [31], is used in order to find subsequence matching in music sequence.

We formally describe the problem of subsequence matching. We describe some relevant definitions for the targeted problem as follows.

Definition 1 (Time Sequence) [32]: A time sequence $S$, $S\left(s_{1}, s_{2}, s_{3}, \ldots, s_{l(S)}\right)$, is an ordered set of real values, where $s_{i}$ is the $i^{t h}$ element of $S$, and $l(S)$ is sequence length of $S$.

Definition 2 (Time Subsequence $\{32]$ : A time subsequence is and ordered sequence. $S_{i, j}=s_{i}, S_{i+1}, s_{i+2}, \ldots, s_{j}$ denotes the time subsequence of a time sequence $S$, which contains the elements of $S$ in position $i$ through $j$ and the length of $S_{i, j}$ is $(l) S_{i, j}=i+j-1$.

Definition 3 (Similan Time Sequence) [32]: Two time $S$ and $Q$ are called similar if and only if $W(S, Q) \geq$, where $W O$ is a function for calculating similarity between $S$ and $Q$, and $\Delta$ is a specified threshold value.

Definition 4-(Similar Sabsequence) [32]: Given two time sequence $S$ and $Q$, the sequences $S^{\prime}$ and $Q^{\prime}$ are called a similar subsequences of $S$ and $Q$ if and only if $S^{\prime}$ and $Q^{\prime}$ are similar and they subsequences of $S$ and $Q$, respectively.

Definition 5 (Distance of Similar Subsequence) [32]: If $S$ and $Q$ have similar subsequences $S_{i, j}$ and $Q_{x, y}$, then the distance between $S_{i, j}$ and $Q_{x, y}$ is $D\left(S_{i, j}, Q_{x, y}\right)=$ $|i-x|$.

Notice that any kind of measure could be used for the similarity function $W()$ in Defintion 3. Based on the above definitions, the shape or trend of two subsequences should be very close if they are similar subsequences.

\subsection{Genre Classification}

Once the genre matching process is performed, we classify the matched music sequence according to music genres. We restrict ourselves to four genres: prehistoric time, middle age, renaissance, baroque, classic and modern, although the approach we use can possibly be extended to much larger classification set. In this paper, we used the decision tree algorithm presented in [33] in order to classify music genres. 
Decision trees are one of the well-known classification techniques. They are widely used due to comparatively rapid to compute, simple to understand by humans, and they can reach accuracies similar to other well-known classification techniques. A decision tree is a tree consisting of a root node, child nodes and edges. Each internal node is a test node that indicates the attribute; the edges indicate the possible values taken on by that attribute. Each non-leaf node consists of a splitting point, and the main task for building the decision tree is to identify the test attribute for each splitting point. The ID3 algorithm uses the information gain to select the test attribute. Information gain can be computed using entropy. In the following, we assume there are $m$ classes in the whole training data set. We know

$$
\operatorname{Entropy}(S)=-\sum_{j=1}^{m} Q_{j}(S) \log Q_{j}(S)
$$

where $Q_{j}(S)$ is the relative frequency of class $j$ in $S$. We can compute the information gain for any candidate attribute $A$ being used to partition $S$ :

$$
\operatorname{Gain}(S, A)-\operatorname{Entropy}(S)-\sum_{v \in A}\left(\frac{\left|S_{v}\right|}{|S|}\right) \operatorname{Entropy}\left(S_{v}\right)
$$

where $v$ represents any possible values of attibutes $A_{i} ; S_{\hat{v}}$ is the subset of $S$ for which attributes $A$ has value $v ;|S|$ is the number of elements in $S$

\section{Conclusion}

In this paper, we have introdyced a study on techniques for automatic music genre recognition and classification. We first Cexplained machine learning based chord recognition methods, suc as hiddem Markov models, neural networks, dynamic Bayesian network and rure-based methods, and template matching methods. We then described supervised, unsuperyised and semi-supervised classification methods classifying music genres. Finally, we briefly introduced the proposed method for automatic classification of musid genres, which consists of three steps: chord labeling using the binarysequence, genre matching using the subsequent match technique and classification of music genres using decision tree.

As for the future work, we plan to implement and describe the proposed method in details. Effectiveness of the proposed method will be compared with various machine learning classification algorithms, including Support Vector Machines and k-nearest neighbor. Through various experiments, we will demonstrate that the proposed method is both fast and accurate comparing to the previously studied methods.

\section{Acknowledgements}

This work was supported by the IT R\&D program of MKE/KEIT. [10041854, Development of a smart home service platform with real-time danger prediction and prevention for safety residential environments].

\section{References}

[1] A. Nasridinov, S. Y. Ihm and Y. H. Park, "A hybrid construction of a decision tree for multimedia contents", Multimedia Tools and Applications, doi: 10.1007/s11042-013-1614-6, (2013).

[2] W. H. Tsai and D. F. Bao, "Clustering Music Recordings Based on Genres", Journal of Information Science and Engineering, vol. 26, (2010). 
[3] A. Sheh and D. P. W. Ellis, "Chord Segmentation and Recognition using EM-Trained Hidden Markov Models", Proceeding of the 4th International Society for Music Information Retrieval Conference (ISMIR), (2003) October 26-30; Baltimore, Maryland, USA.

[4] J. P. Bello and J. Pickens, (Eds.), "A Robust Mid-level Representation for Harmonic Content in Music Signals”, Proceeding of the 6th International Society for Music Information Retrieval Conference (ISMIR), (2005) September 11-15; London, UK.

[5] K. Lee and M. Slaney, "Automatic Chord Recognition from Audio Using an HMM with Supervised Learning", Proceeding of the 7th International Society for Music Information Retrieval Conference (ISMIR), (2006) October 8-12; Victoria, BC, Canada.

[6] K. Lee and M. Slaney, "Acoustic Chord Transcription and Key Extraction From Audio Using Key-Dependent HMMs Trained on Synthesized Audio", IEEE Transactions on Audio, Speech, and Language Processing, vol. 16, no. 2, (2008).

[7] H. Papadopoulos and G. Peeters, "Simultaneous Estimation of Chord Progression and Downbeats from an Audio File", Proceeding of the 33th International Conference on Acoustics, Speech, Signal Processing (ICASSP), (2008) March 31-April 4; Las Vegas, USA.

[8] G. Peeters, "Template-Based Estimation of Time-Varying Tempo", EURASIP Journal on Adyances in Signal Processing, Article ID 67215, (2007).

[9] M. Khadkevich and M. Omologo, "Use of Hidden Markov Models and Factored Language Models for Automatic Chord Recognition", Proceeding of the 10th International Society for Music Information Retrieval Conference (ISMIR), (2009) October 26-30; Kobe, Japan

[10] R. Chen, W. Shen, A. Srinivasamurthy and P. Chordia, "Chord Recognition Using Duration-Explicit Hidden Markov Models", Proceeding of the 14th International Society for Music Information Retrieval Conference (ISMIR), (2013) November 4-8; Curitiba, Brazil.

[11] C. Taemin and P. B. Juan, "Real-time implementation of hmm-based chord estimation in musical audio", Proceedings of the International Computer Music Conference (ICMC), (2009) August 16-21; Montreal, Canada.

[12] J. Osmalskyj, J. J. Embrechts, S. Piérard-and M. Van Proogenbroeck, "Neural Networks for Musical Chords Recognition", Journees D’Informatiotique Musicale, (2012) May 9-11; Mons, Belgium.

[13] N. Boulanger-Lewandowski, Y. Bengio and P. Vjncent, "Audio Chord Recognition with Recurrent Neural Networks", Proceeding of the 14th International Society for Music Information Retrieval Conference (ISMIR), (2013) November 4-8, Guritiba, Brazi.

[14] M. Mauch, K. Noland_and(S. Dixon, "Using Musical Structure to Enhance Automatic Chord Transcription", Proceeding of the 10th Inteynational Sqciety for Music Information Retrieval Conference (ISMIR), (2009) October 26-30; Kobe Japan.

[15] M. Mauch, (Ed.) "Automatic Chord Transcription from Audio Using Computational Models of Musical Context", PhD Thesis, Queen Mary, University of London, (2010).

[16] M. Mauch ands. Dixon, "Simultaneous Estimation of Chords and Musical Context from Audio", IEEE Transactions on Audio, Speech and Language Processing, vol. 18, no. 6, (2010).

[17] A. Shenoy and Y. Wang, Key, Chord, and Rhythm Tracking of Popular Music Recordings", Journal Computer Music, vol. 29, ho. 3, (2005).

[18] C. Sailer and K. Rosenbauer, "A Bottom-Up Approach to Chord Detection", Proceeding of the International Computer Music Conference, (2006) San Francisco, USA.

[19] L. Oudre, Y Grenier and C. Fevotte, "Chord Recognition Using Measures of Fit, Chord Templates and Filtering Methods", Proceeding of the 34th International Conference on Acoustics, Speech, Signal Processing (ICASSP, (2009) October 18-21; New Paltz, NY, USA.

[20] L. Oudre, Y. Grenier and C. febotte, "Chord Recognition by Fitting Rescaled Chroma Vectors to Chord Templates", IEEE Transactions on Audio, Speech, and Language Processing, vol. 19, no. 7, (2011).

[21] L. Oudre, Y. Grenier and C. Fevotte, "Template-based Chord Recognition: Influence of the Chord Types", Proceeding of the 10th International Society for Music Information Retrieval Conference (ISMIR), (2009) October 26-30; Kobe, Japan.

[22] L. Oudre, C. Fevotte and Y. Grenier, "Probabilistic Template-Based Chord Recognition", IEEE Transactions on Audio, Speech, and Language Processing, vol. 19, no. 8, (2011).

[23] L. Oudre, C. Fevotte and Y. Grenier, "Probabilistic framework for template-based chord recognition", Proceeding of International Workshop on Multimedia Signal Processing (MMSP), (2010) October 4-6; Saint Malo, France.

[24] K. Lee, "Automatic Chord Recognition from Audio Using Enhanced Pitch Class Profile", Proceeding of the International Computer Music Conference, (2006) New Orleans, USA.

[25] C. Xu, M. C. Maddage, X. Shao and F. Cao, "Musical genre classification using support vector machines", Proceeding of International Conference on Acoustic, Sppech, and Signal Processing, (2003). 
[26] G. Tzanetakis and P. Cook, "Musical Genre Classification of Audio Signals", IEEE Transactions on Audio, Speech, and Language Processing, vol. 10, no. 5, (2002).

[27] J. Wülfing and M. Riedmiller, "Unsupervised Learning of Local Features for Music Classification", Proceedings of the 13th International Society for Music Information Retrieval Conference, (2012) October 812; Porto, Portugal.

[28] X. Shao, C. Xu and M. S. Kankanhalli, "Unsupervised classification of music genre using hidden Markov model", Proceeding of International Conference on Multimedia and Expo, (2004) June 27-30.

[29] Y. Xu, C. Zang and J. Yang, "Semi-Supervised Classification of Musical Genre Using Multi-View Features", Proceeding of International Conference on Computer Music, (2005).

[30] S. Brecheisen, H. P. Kriegel, P. Kunath and A. Pryakhin, "Hierarchical Genre Classification for Large Music Collections", Proceeding of International Conference on Multimedia and Expo, (2006) July 9-12; Toronto, Ont., Canada.

[31] S. Y. Ihm, A. Nasridinov, J. H. Lee and Y. H. Park, "Efficient duality-based subsequent matching on timeseries data in green computing", Journal of Supercomputing, DOI: 10.1007/s11227-013-1028-2, (2013).

[32] V. S. Tseng, L. C. Chen and J. J. Liu, "Gene relation discovery by mining similar subsequence in timeseries microarray data", Proceedings of the IEEE symposium on computational intelligence in bioinformatics and computational biology (CIBCB), (2007).

[33] A. Nasridinov, Y. S. Lee and Y. H. Park, "Decision Tree Construction on GPU: Ubiquitous Parallel Computing Approach", Computing, vol. 96, no. 5, (2013).

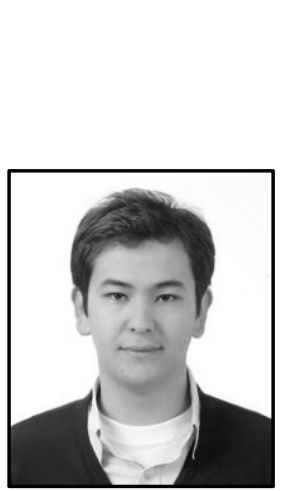

Aziz Nasridinov

\section{Authors}

Aziz Nasridinan received his B.S. degree in Computer Science from Tashkent University of Information Technology and his M.S. and Ph.D. degrees in Computer Entgineering from Dongguk University, South Korea. He is currently working as Research Professor at Dongguk University at Gyeongju, South Korea. His research interests include Database Management Systems (DBMS), machine-learning techniques and Web Services.

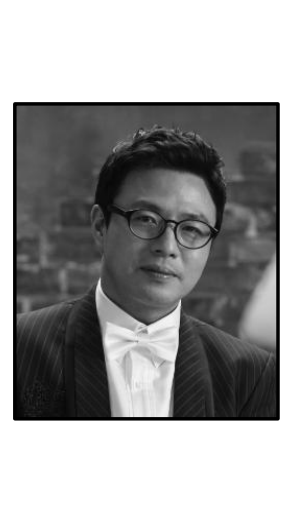

\section{Young-Ho Park}

Young-Ho Park is an Associate Professor of the Multimedia Science at Sookmyung Women's University. His research interests include Database Management Systems (DBMS), Information Retrieval (IR), XML, and Telecommunication Systems. Young-Ho Park received his Ph.D. degree in Department of Computer Science from the Korea Advanced Institute of Science and Technology ( KAIST ) in 2005 . His Ph.D. research includes efficient query processing in heterogeneous XML documents. He received his B.S. and M.S. degrees in Computer Engineering from the Dongguk University in 1990 and 1992. He had worked for the Electronics and Telecommunication Research Institute ( ETRI ) as a senior research staff at the ISDN Administration \& Maintenance Division for TDX-10 ISDN, the Real-Time DBMS Division and the Real-Time Operating System Division from 1993 - 1999. And, he had worked for the Advanced Information Technology Research Center ( AITrc ), Korea Advanced Institute of Science and Technology ( KAIST ) as a Post Doctor at the from 2005 - 2006 after receiving Ph.D. degree. 
International Journal of Multimedia and Ubiquitous Engineering Vol.9, No.4 (2014)

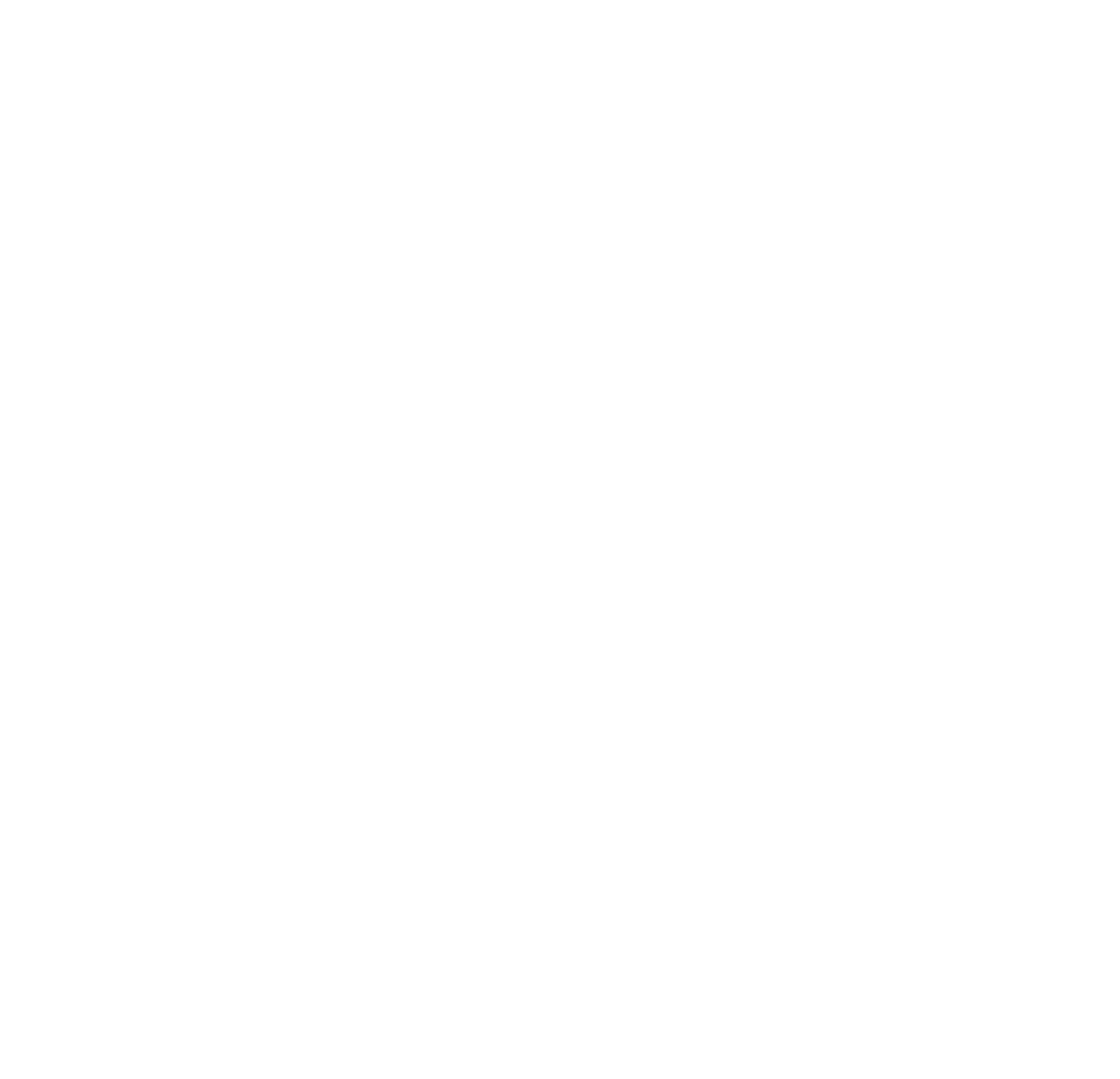

